\title{
Review of: "Deficiency of intestinal Bmal1 prevents obesity induced by high-fat feeding"
}

\author{
$\mathrm{Cu}^{1}$ \\ 1 4th Military Medical University
}

Potential competing interests: The author(s) declared that no potential competing interests exist.

Review of: Deficiency of intestinal Bmal1 prevents obesity induced by high-fat feeding Mingkai Li and Di Qv

Department of Pharmacology, School of Pharmacy, the Fourth Military Medical University

In this research, the authors proved that the Bmal1 played an important role in the energy homeostasis, down-regulation of Bmal1 could protect the animal from obesity and related abnormalities such as hyperlipidemia. Additionally, the mechanism exploration was conducted and the results revealed that the Bmal1 promoted dietary fat absorption via regulating Dgat2 transcription and expression. Inhibiting of intestinal Bmal1 protected mice from HFD-induced obesity. All the findings in this research suggested that intestinal BMAL1 might be a drug target for management of metabolic diseases induced by excess fat intake.

However, there are still two suggestions for this present research:

1. The loss of Rev-erb $\alpha$ affected the index of weight, fat mass, circulating lipids, the lipids in the intestine and the lipid metabolites in the liver (including triglycerides, free fatty acids and lipid droplets). At the same time, the expression level of Dgat2 was increased, indicating that the lack of Rev-erba aggravated the obesity phenotype. If other molecules related to lipid metabolism can be tested in this research, including triglyceride synthesis, free fatty acid uptake, lipid droplet formation, lipid oxidation, and chylomicron packaging, it will provide the reader a more comprehensive picture.

2. This study focused on the changes in the metabolic phenotype after the knockout or activation of bmal1 and Rev-erba in the small intestine, it will be more significant to observe the changes in the function on circadian rhythm and secretory factors in the small intestine. While in the study of obesity induced by HFD, more data in the glucose metabolism and insulin resistance will be interesting exclude the lipid metabolism. 